NOTA GEOLÓGICA

\title{
La granodiorita orbicular del Cordón de Lila, región de Antofagasta, Chile
}

\author{
Hans Niemeyer Rubilar ${ }^{1}$
}

1 Departamento de Ciencias Geológicas, Universidad Católica del Norte, Avda. Angamos 0610, Casilla 1280, Antofagasta, Chile. hansn@ucn.cl

RESUMEN. La granodiorita orbicular del Cordón de Lila se emplaza dentro del plutón ordovícico El León, en el flanco suroriental del Cordón de Lila. Esta conforma una pequeña pipa hospedada en una banda de aplita en contacto con un roof-pendant de gabro de anfíbola. La presencia de allanita, considerado un mineral trazador de los procesos de cristalización magmática, en las tres facies que constituyen la pipa (interior, borde de las orbículas y matriz), permite sugerir que las orbículas de esta granodiorita son el resultado del proceso de cristalización magmática que afectó al magma que dio origen a la granodiorita orbicular.

Palabras clave: Granodiorita orbicular, Orbículas magmáticas.

ABSTRACT. The orbicular granodiorite from Cordón de Lila, Antofagasta Region, Chile. The orbicular granodiorite of the Cordón de Lila is emplaced within the Ordovician pluton El León, in the southeastern part of Cordón de Lila. This forms a small pipe emplaced in an aplitic band, near the contact with a roof-pendant of amphibole bearing gabbro. The presence of allanite, considered a tracer mineral in magmatic crystallization processes, in the three facies that constitute the pipe (core, margin and matrix) suggests that the orbicules of this granodiorite are the result of magmatic crystallization that affected the magma that originated the orbicular granodiorite. 


\section{Introducción}

Los granitoides orbiculares, aunque raros y de ocurrencia local, han recibido desde larga data la atención de los petrólogos (Bäckström, 1893; Johannsen, 1932; Leveson, 1963) debido a su espectacular envolvente concéntrica en torno a un núcleo formado ya sea por fragmentos sólidos (xenolitos) o por material magmático de otra índole (Leveson, 1966). El origen de las orbículas es motivo de controversia entre aquellos autores que sostienen que los bordes de las orbículas son de origen ígneo (Enz et al., 1979) y quienes aducen un origen metasomático (Thompson y Giles 1974, 1980). Sin duda, no existe una explicación única para todos los granitoides orbiculares y en la presente nota se da a conocer un tipo especial de granodiorita orbicular, diferente a los descritos anteriormente (Aguirre et al., 1974; Leveson, 1966; Godoy, 1997), con énfasis en el origen del borde de las orbículas.

\section{Descripción del Afloramiento}

La Granodiorita orbicular del Cordón de Lila (Fig. 1) conforma una pequeña pipa de $10 \mathrm{~m}$ de largo por $5 \mathrm{~m}$ de ancho en el extremo meridional del Plutón El León, de edad ordovícica (467,1×3,3 Ma;
Niemeyer et al., 2014). Esta pipa intruye un cuerpo de aplita de $150 \mathrm{~m}$ de ancho elongado en sentido este-oeste, en el contacto sur de un roof-pendant de gabro de anfíbola que cubre una superficie aproximada de 0,3-0,4 km² dentro del Plutón El León (Fig. 2). $\mathrm{Al}$ interior del gabro se observan varios apófisis de granodiorita idéntica a la que contiene orbículas, pero carentes de ellas. Solo la pipa emplazada en la aplita contiene orbículas. Estas son de un diámetro máximo de $10-15 \mathrm{~cm}$ con forma esférica a ovalada (Fig. 3). Las orbículas ovaladas aumentan en número de sur a norte dentro de la pipa, según una orientación subparalela a la foliación cercanamente este-oeste de la granodiorita. En el extremo norte, las orbículas entran en contacto entre sí, dejando entre ellas solo un pequeño porcentaje de matriz. En este caso, la forma ovalada de las orbículas se debe precisamente a la interacción entre ellas, que las obliga a cambiar de forma por apilamiento del material magmático en proceso de flujo paralelo a la elongación mayor de la pipa. La parte central de las orbículas está constituida por una granodiorita de textura hipidiomorfa de grano fino a medio (2-3 $\mathrm{mm})$, en tanto que la matriz está formada por el mismo tipo de granodiorita pero de grano más grueso $(4-5 \mathrm{~mm})$.

La granodiorita presenta textura hipidiomorfa de grano medio y está compuesta por plagioclasa, algo

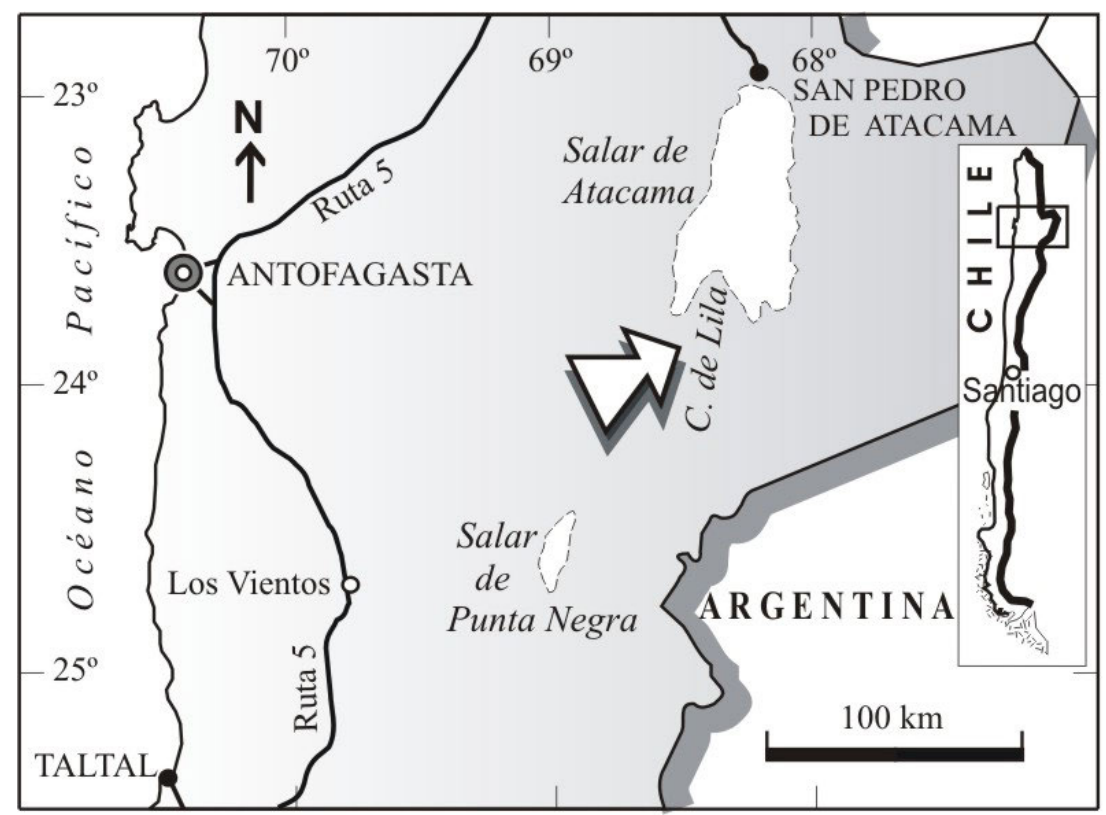

FIG. 1. Ubicación del Cordón de Lila en el norte de Chile. 


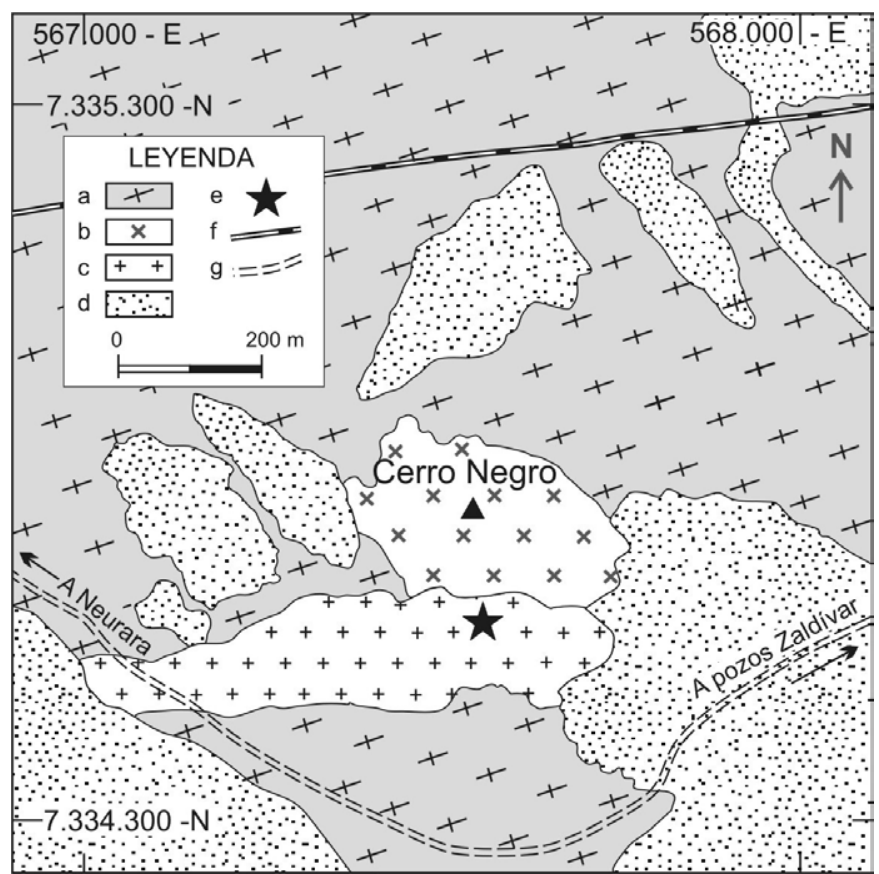

FIG. 2. Ubicación de la pipa de granodiorita orbicular, en el extremo suroriental del Plutón El León, Cordón de Lila. a. Granodiorita El León (Ordovícico); b. Roof-pendant de gabro de anfíbola de Cerro Negro (¿Ordovícico?); c. Banda de aplita (¿Ordovícico?); d. Brechas volcánicas y depósitos aluviales (Cuaternario); e. Ubicación de la pipa de granodiorita orbicular; f. Acueducto; g. Camino. Modificado de Carrasco (2015).

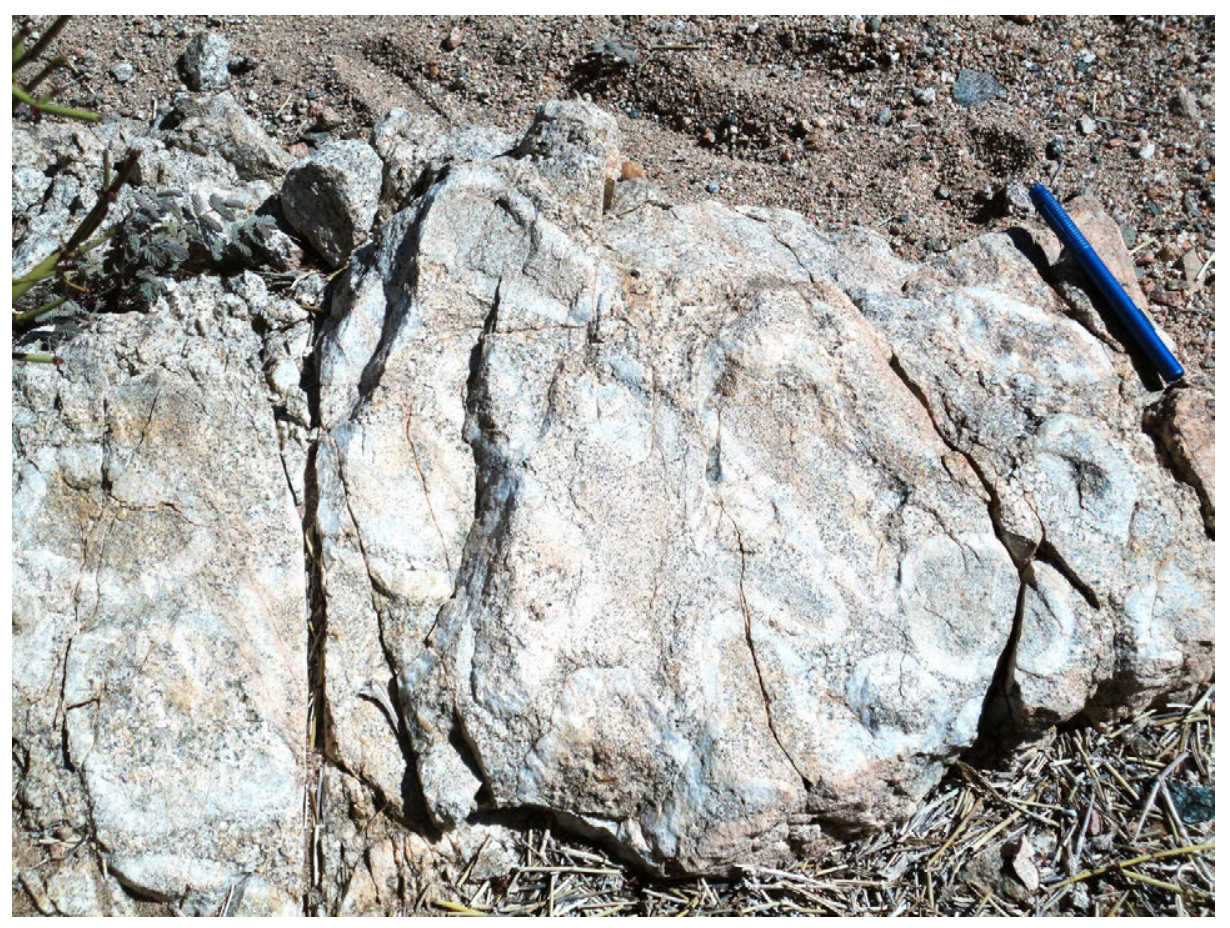

FIG. 3. Orbículas en la pipa de granodiorita orbicular del Cordón de Lila. 
de feldespato alcalino, cuarzo, biotita, muscovita y allanita. El borde felsítico de las orbículas tiene alrededor de $1 \mathrm{~cm}$ de ancho y presenta un color blanco sin estructura macroscópica concéntrica o radial, como en la mayoría de los casos citados en la literatura (Aguirre et al., 1974; Leveson, 1966; Godoy, 1997). Los bordes son de grano muy fino, compuestos por plagioclasa y cuarzo. Su contacto interior es transicional con la parte central, en tanto que su contacto externo con la matriz es tajante $y$, en algunos casos, marcado por una capa de aproximadamente $1 \mathrm{~mm}$ de espesor constituida por biotita de grano fino. Un aspecto notorio de estas orbículas respecto a otras descritas en la literatura es su carencia de un núcleo interno, ya sea este un megacristal o un xenolito (Leveson, 1966). Además, su borde carece totalmente de estructura interna, ya sea radial o concéntrica (Leveson, 1966), y se presenta, macroscópicamente, como una masa homogénea, maciza, sin estructura interna. Solo en algunos bordes se observa estructura radial al microscopio (Fig. 4).

\section{Discusión}

La cristalización de biotita y muscovita dentro de la granodiorita de la pipa se explica por un aumento de la presión de agua (Maaloe y Wyllie, 1975) durante la última etapa de cristalización del magma que la conforma.

Dos hipótesis son plausibles para que las orbículas se formen tan regularmente en torno al material granodiorítico. Una, autóctona, en que las orbículas se habrían producido in situ como parte del proceso de cristalización del magma, denominado magma orbicular (Decitre et al., 2002), es decir, tendrían un origen netamente magmático. La otra, alóctona, en que el material granodiorítico migraría hasta un lugar en el borde norte de la pipa donde habría encontrado un fluido félsico envolvente (origen metasomático).

Para las orbículas del Plutón El León se sugiere que ocurrió una cristalización breve de material félsico en cierto momento del desarrollo de la cristalización del magma granodiorítico. El contacto abrupto entre el borde de las orbículas y el material de la matriz sugiere que hubo un súbito cambio en el proceso de cristalización, que puso fin a la separación de la facies félsica y permitió la cristalización de la matriz. Este argumento es válido para la primera hipótesis de formación de las orbículas. Aún más, la presencia de allanita en las tres facies que conforman la pipa (interior, borde de las orbículas y matriz envolvente) sugiere que ellas se formaron a partir de un mismo magma. En efecto, el mineral allanita ha sido considerado como un trazador de los procesos de cristalización magmática (Hoshino et al., 2007).

Al respecto, se sostiene que el borde de las orbículas requiere de un sobrecalentamiento del magma parental para su formación (Vernon, 1985). Dicho sobrecalentamiento sería necesario para destruir la formación de núcleos de cristalización, produciendo el material félsico de grano fino que constituye los bordes por un intervalo breve de tiempo. El agua contenida en el magma félsico provocaría el sobrecalentamiento del mismo, por disminución de la temperatura del liquidus (Vernon, 1985). Además, se produciría un gradiente termal entre el material félsico y la parte central de las orbículas, que permitiría una termodifusión por el efecto Soret, de tal manera que $\mathrm{Si}, \mathrm{K}, \mathrm{Na}$ y Al migrarían hacia el borde de las orbículas, en tanto que $\mathrm{Ca}, \mathrm{Fe}$, Ti y Mg migrarían en sentido inverso (Sylvester, 2011).

La ausencia de núcleos de cristalización retardaría, además, la cristalización permitiendo un grado significativo de sobre-enfriamiento final, lo cual obligaría a que la cristalización del material de grano fino del borde de las orbículas se lleve a cabo (Vernon, 1985). La falta notable de estructura interna en la mayoría de los bordes de las orbículas indica condiciones de presión y temperatura uniformes durante su formación.

\section{Conclusiones}

Se considera en este trabajo que la presencia de allanita en las tres facies que conforman la pipa (interior, borde de las orbículas y matriz envolvente) constituye un trazador de la cristalización de las orbículas a partir de un magma granodiorítico único, lo cual favorece un origen magmático para las orbículas. Se concluye, además, que para la formación de las orbículas no es necesaria la presencia de un núcleo interno que catalice la cristalización del magma.

\section{Agradecimientos}

Las observaciones de W. Vivallo, E. Godoy y de un evaluador anónimo contribuyeron a mejorar la presente nota. A. Eade de GABGEO confeccionó las microfotografías. Los dibujos se deben a L. Jofré, del Departamento de Ciencias Geológicas de la Universidad Católica del Norte. 

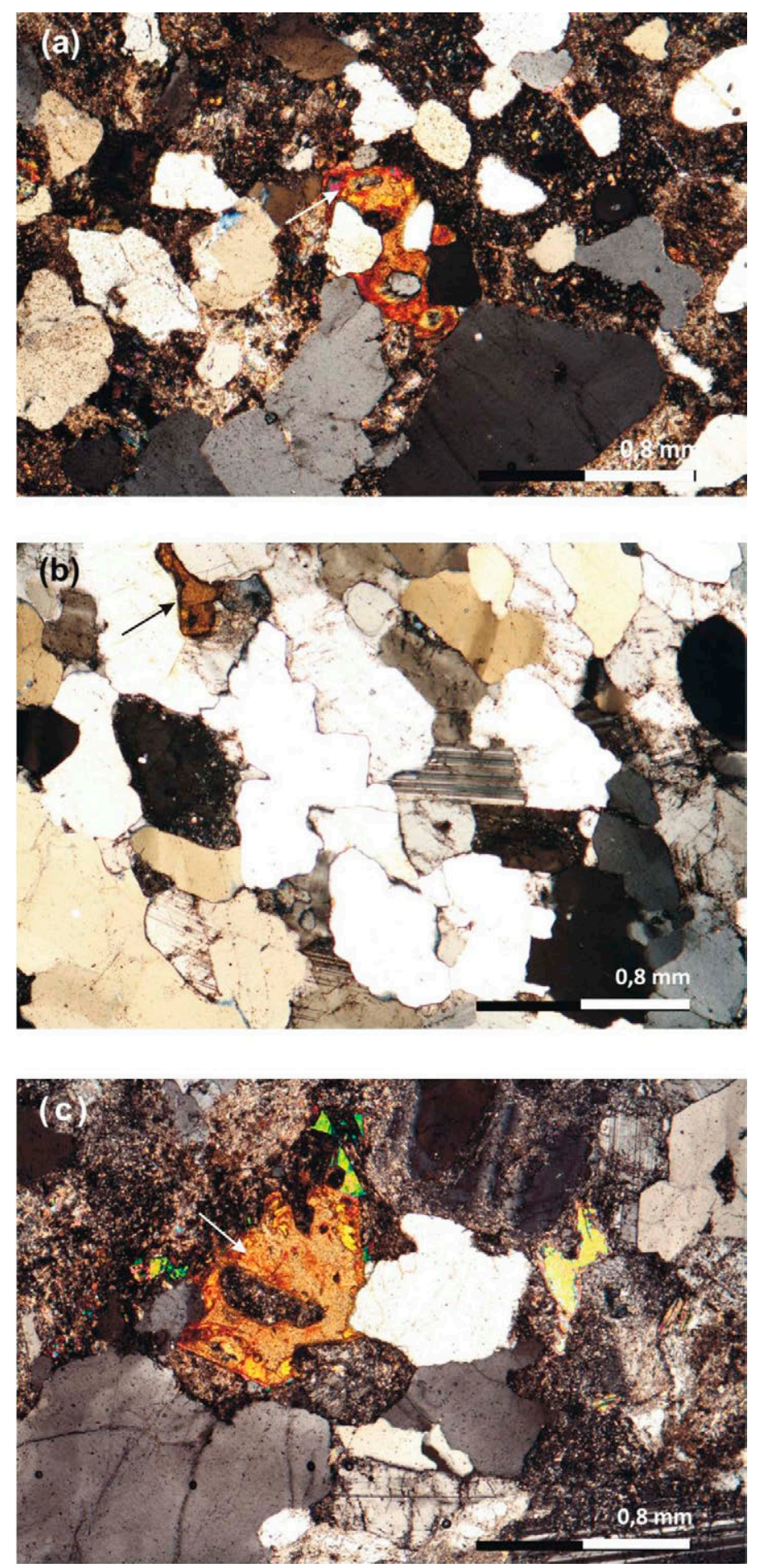

FIG. 4. Microfotografías de las tres facies que conforman la pipa de la granodiorita orbicular, que muestra cristales de allanita a nícoles cruzados. a. Interior con textura hipidiomorfa de grano fino a medio; b. Borde de grano fino con orientación subparalela de cristales de cuarzo y plagioclasa; c. Matriz envolvente con textura hipidiomorfa de grano grueso. En las tres microfotografías se indican cristales de allanita mediante una flecha. 


\section{Referencias}

Aguirre, L.; Hervé, F.; Del Campo, M. 1974. An orbicular tonalite from Caldera, Chile. Journal Faculty of Science, Hokkaido University IV, 17 (2): 231-259.

Bäckström, H. 1893. Causes of magmatic differentiation. Journal of Geology 1: 773-779.

Carrasco, J.L. 2015. La granodiorita El León, extremo sureste del Cordón de Lila, Región de Antofagasta, Chile. Memoria de título (Inédito), Universidad Católica del Norte: 52 p.

Decitre, S.; Gasquet, D.; Marignac, Ch. 2002. Genesis of orbicular granitic rocks from the Ploumanac'h Plutonic Complex (Brittany, France): petrographical, mineralogical and geochemical constraints. European Journal of Mineralogy 14: 715-731.

Enz, R.D.; Kudo, A.M.; Brookins, D.G. 1979. Igneous origin of the orbicular rocks of the Sandia Mountains, New Mexico: Summary. Geological Society of America Bulletin, Part I 90: 138-140.

Godoy, E. 1997. El gabro orbicular de quebrada Ánimas Viejas, Provincia de Chañaral, Chile. In Congreso Geológico Chileno, No. 8, Actas II: 1285-1289. Antofagasta.

Hoshino, M.; Kimata, M.; Shimizu, M.; Nishida, N. 2007. Minor-element systematics of fluorapatite and zircon inclusions in allanite-(Ce) of felsic volcanic rocks from three orogenic belts: Implications for the origin of their host magmas. The Canadian Mineralogist 45 (6): 1337-1353.

Johannsen, A. 1932. A descriptive petrography of the igneous rocks, Volume II, University of Chicago Press: 248-259.
Leveson, D.J. 1963. Orbicular rocks rocks of the Lonesome Mountain area, Beartooth Mountains, Montana, and Wyoming. Geological Society of America Bulletin 74: $1015-1040$

Leveson, D.J. 1966. Orbicular rocks: A review. Geological Society of America Bulletin 77: 409-426.

Maaloe, S.; Wyllie, P.J. 1975. Water content of a granite magma deduced from the sequence of crystallization determined experimentally with water-undersaturated conditions. Contributions to Mineralogy and Petrology 52: 175-191.

Niemeyer, H.; Meffre, S.; Guerrero, R. 2014. Zircon U-Pb geochronology of granitic rocks of the Cordón de Lila and Sierra de Almeida ranges, northern Chile: $30 \mathrm{~m} . \mathrm{y}$. of Ordovician plutonism on the western border of Gondwana. Journal of South American Earth Sciences 56: 228-241.

Sylvester, A.G. 2011. The nature and polygenic origin of orbicular granodiorite in the Lower Castle Creek pluton, northern Sierra Nevada batholith, California. Geosphere 7 (5): 1-9.

Thompson, T.B.; Giles, D.L. 1974. Orbicular rocks of the Sandia Mountains, New Mexico. Geological Society of America Bulletin 85: 911-916.

Thompson, T.B.; Giles, D.L. 1980. Discussion of "Igneous origin of the orbicular rocks of the Sandia Mountains, New Mexico". Geological Society of America Bulletin 91: 245-246.

Vernon, R. 1985. Possible role of superheated magma in the formation of orbicular granitoids. Geology 13: $843-845$.

Manuscript received: May 23, 2017; revised/accepted: October 3, 2017; available online: October 3, 2017. 